\title{
IgG subclass antibodies to mycobacterial sonicate and recombinant antigens in leprosy
}

\author{
R. SHEELA, ${ }^{*} \ddagger$ N. P. SHANKERNARAYAN, $\dagger$ \\ G. RAMU $\dagger \&$ V. R. MUTHUKKARUPPAN* \\ *Department of Immunology, School of Biological Sciences, \\ Madurai Kamaraj University, Madurai, 625 021, India, \\ $\dagger$ Voluntary Health Services, Leprosy Project, Sakthinagar, 638 \\ 315 , India
}

Accepted for publication 3 June 1994

\begin{abstract}
Summary In this study the IgG subclass antibodies to soriicated preparations of Mycobacterium leprae (leprosin A) and BCG (BCG-S) as well as to purified recombinant $65 \mathrm{kDa}$ protein of $M$. leprae (rML65) were analysed in sera from leprosy patients and healthy household contacts (HFC) and noncontacts (HNC) in a leprosy endemic population. In LBI + (lepromatous bacterial index positive) patients, IgG3 was predominant in the responses to sonicated antigens of $M$. leprae. Following chemotherapy, IgG3 responses were reduced while IgG2 levels were increased. On the other hand, IgG response to rML65 was dominated by IgG1 in all the patient and control groups. Interestingly, the level of antileprosin A IgG antibody in erythema nodosum leprosum (ENL) was similar to that of lepromatous groups, while the level of anti-rML65 IgG antibody was significantly reduced in ENL. IgG4 antibodies to the antigens studied were only at low levels in all groups, including ENL. Significant differences were observed between HNC and HFC in the pattern of IgG subclass antibodies to sonicated antigens, even though their antigen specific IgG levels were similar. While HNC showed equivalent proportion of $\mathrm{IgG} 1$ and $\mathrm{IgG} 2$ in their responses to leprosin $\mathrm{A}$ and BCG-S, HFC showed a specific increase in IgG1 levels, suggesting that both groups are distinctly different. Further studies are required to elucidate the functional significance of $\mathrm{IgG}$ subclass pattern in pathogenesis and the mechanism of immunoregulation resulting in the high levels of $\mathrm{IgG} 1$ and $\mathrm{IgG} 3$ antibodies to $M$. leprae protein antigens in lepromatous leprosy.
\end{abstract}

\section{Introduction}

Leprosy, a chronic infectious disease caused by Mycobacterium leprae, presents a wide immunological spectrum. At one end, tuberculoid leprosy patients mount a good cell-

$\ddagger$ Correspondence: Sheela Ramanathan, Inserm U28, Hopital Broussais, 96 rue Didot, 75674, Paris, CEDEX 14, France. 
mediated immune response and contain the disease. ${ }^{1,2}$ At the other end, lepromatous patients exhibit a specific T-cell anergy ${ }^{3}$ and have elevated serum IgG levels compared to normal individuals. ${ }^{4-6}$ Antibodies to $M$. leprae antigens occur in increasing amounts from the tuberculoid to the lepromatous end of the spectrum. ${ }^{7,8}$ However, the subclasses of these antibodies have not been analysed in detail except in the report of Dhandayuthapani et al. ${ }^{9}$

The 4 different IgG subclasses have different effector functions and appear to be differently regulated in humans. ${ }^{10}$ Antibodies to protein antigens predominantly belong to IgG1, IgG3 or IgG4, while polysaccharide antigens preferentially elicit IgG2 antibodies. ${ }^{11,12}$ IgG2 and IgG4 are inefficient in fixing complement. ${ }^{13}$ Specific subclasses of IgG have been observed to become predominant after certain bacterial, ${ }^{12}$ viral $^{14,15}$ and parasitic ${ }^{16,17}$ infections. Expression of different Ig subclasses is regulated by cytokines. While this regulation is well documented in the murine system, ${ }^{18}$ little is known about it in humans. ${ }^{19}$ This study was aimed at analysing the subclasses of IgG antibodies to sonicate antigens of $M$. leprae and BCG as well as to recombinant $M$. leprae $65 \mathrm{kDa}$ protein in leprosy patients and endemic controls.

\section{Materials and methods}

SERA

Blood samples were obtained from leprosy patients attending a leprosy hospital located at Sakthinagar in Periyar District, about $300 \mathrm{~km}$ from Madurai. The hospital, which is managed by Sakthi Sugars Limited, is a leprosy control unit under the Tamil Nadu Government Health Services. Leprosy patients were diagnosed clinically and the bacterial index (BI) was determined in all patients by the slit-skin smear test. The patients were classified according to clinical criteria into 5 groups: polar lepromatous (LL), borderline lepromatous (BL), borderline tuberculoid (BT) and polar tuberculoid (TT). ${ }^{20}$ Both untreated cases and patients treated for varying lengths of time were included. $\mathrm{LL}$ and BL were grouped together and segregated into BI positive $(\mathrm{LBI}+)$ and BI negative (LBI-) lepromatous patients. Samples were collected from ENL patients at the time of reactions. Household contacts of leprosy patients (healthy family contacts, HFC) were thoroughly examined for leprosy lesions before taking blood samples. Leprosy patients and their family contacts were selected randomly without any bias towards sex. Their age varied from 15 to 60 years. Healthy noncontacts (HNC) were members of the staff and students of the School of Biological Sciences, Madurai Kamaraj University, who have not had any known habitual contact with leprosy patients even though they live in leprosy endemic area. Both sexes were included and their age varied from 21 to 40 years. All blood samples were collected with prior consent.

\section{ANTIGENS}

Leprosin A (batch CD141) was obtained from Dr R. J. W. Rees (WHO/UNDP/ IMMLEP $M$. leprae bank). The sonicate antigen was prepared from armadillo derived $M$. leprae by ultrasonication. After centrifugation at $12,000 \times \mathbf{g}$ the supernatant was filtered through a $0 \cdot 22 \mu \mathrm{M}$ membrane filter and the protein concentration was adjusted to $1 \mathrm{mg} / \mathrm{ml}$. Aliquots were stored at $-70^{\circ} \mathrm{C}$ as recommended. $M$. bovis BCG sonicate 
(BCG-S) was prepared from $1 \mathrm{~g}$ of bacilli following the procedure used for leprosin $\mathrm{A}$ preparation. Recombinant $M$. leprae $65 \mathrm{kDa}$ (rML65) antigen was generously supplied by Dr Van Embden, Bilthoven, The Netherlands through the UNDP/World Bank/ WHO Special Programme for Research and Training in Tropical Diseases. The freezedried material was reconstituted with Dulbecco's phosphate-buffered saline at a concentration of $1 \mathrm{mg} / \mathrm{ml}$.

\section{ESTIMATION OF IgG SUBCLASS ANTIBODIES TO BCG-S, LEPROSIN A AND rML65}

IgG subclass levels to BCG-S, leprosin A and eML65 were determined by ELISA using monoclonal antibody (MoAb) reagents from Boehringer Mannheim GmnH (Germany). Briefly, Nunc flat bottomed microtitre plates were coated with $50 \mu \mathrm{l} /$ well of antigens at appropriate concentration for $1 \mathrm{~h}$ at $37^{\circ} \mathrm{C}$ and overnight at $4^{\circ} \mathrm{C}$. After blocking with $1 \%$ gelatin, pooled lepromatous serum (LSP) or the serum samples were added at a dilution of $1: 20$. The plates were incubated for $1 \mathrm{~h}$ at $37^{\circ} \mathrm{C}$. MoAb directed against the human IgG subclasses (IgG1 (clone NL16), IgG2 (clone GOM1), IgG3 (clone ZG4) and IgG4 $(\text { clone RJ4) })^{21,22}$ were added at a dilution of $1: 500(50 \mu \mathrm{l} /$ well $)$ and incubated at $37^{\circ} \mathrm{C}$ for $2 \mathrm{~h}$ followed by $50 \mu \mathrm{l}$ of peroxidase conjugated rabbit antimouse IgG (1:2000) (Cappel; preadsorbed with human Ig) and incubated for $1 \mathrm{~h}$ at $37^{\circ} \mathrm{C}$. In parallel, 1 set of plates were treated with goat antihuman IgG $(1: 2000)$ for $1 \mathrm{~h}$. Subsequently the reaction was developed with OPD substrate.

Optimal dilution for MoAb against the human IgG subclasses was 1:500. Optimal coating concentration of the antigens and serum dilution was determined by checker board titration. BCG-S, Leprosin A and rML65 were coated at a concentration of $4 \mu \mathrm{g} /$ $\mathrm{ml}$ and the serum samples were used at a dilution of $1: 20$. LSP was included every time ELISA was performed and the coefficient of variation for 6 separate assays was $5 \cdot 2 \%$. Individual IgG subclass response was expressed as a percentage of the total of absorbance values for the 4 subclasses. ${ }^{23}$ Mean +2 SD of HNC was used as cut-off value to determine the percentage of positivity.

\section{Results}

The levels of IgG antibodies against BCG-S and leprosin A were significantly elevated in lepromatous patients, compared to HFC, HNC and TT/BT groups $(p<0.05$; Figure 1$)$. However, they were similar among LBI + and LBI- groups. Antileprosin A IgG response in ENL was comparable with lepromatous patients, while the level of antirML65 IgG antibody was significantly reduced in ENL. With regard to rML65 specific IgG antibodies, LBI + patients had the highest and ENL the lowest levels and the difference $(p<0.05)$ was significant. Further, with the reduction in bacterial load, there was concomitant decrease in the level of IgG antibodies to rML65.

Several major differences were observed in the pattern of IgG subclass antibodies to leprosin A and BCG-S among different groups of patients and controls. The proportion of antileprosin A IgG3 antibody was significantly higher in the LBI + than in the LBIgroup $(p<0.05$; Table 1). This increase was also reflected in the percentage of individuals with higher IgG3 response (mean $+2 \mathrm{SD}$ of $\mathrm{HNC}$; Table 2). Following chemotherapy, IgG3 levels were decreased with a concomitant increase in antibodies of 

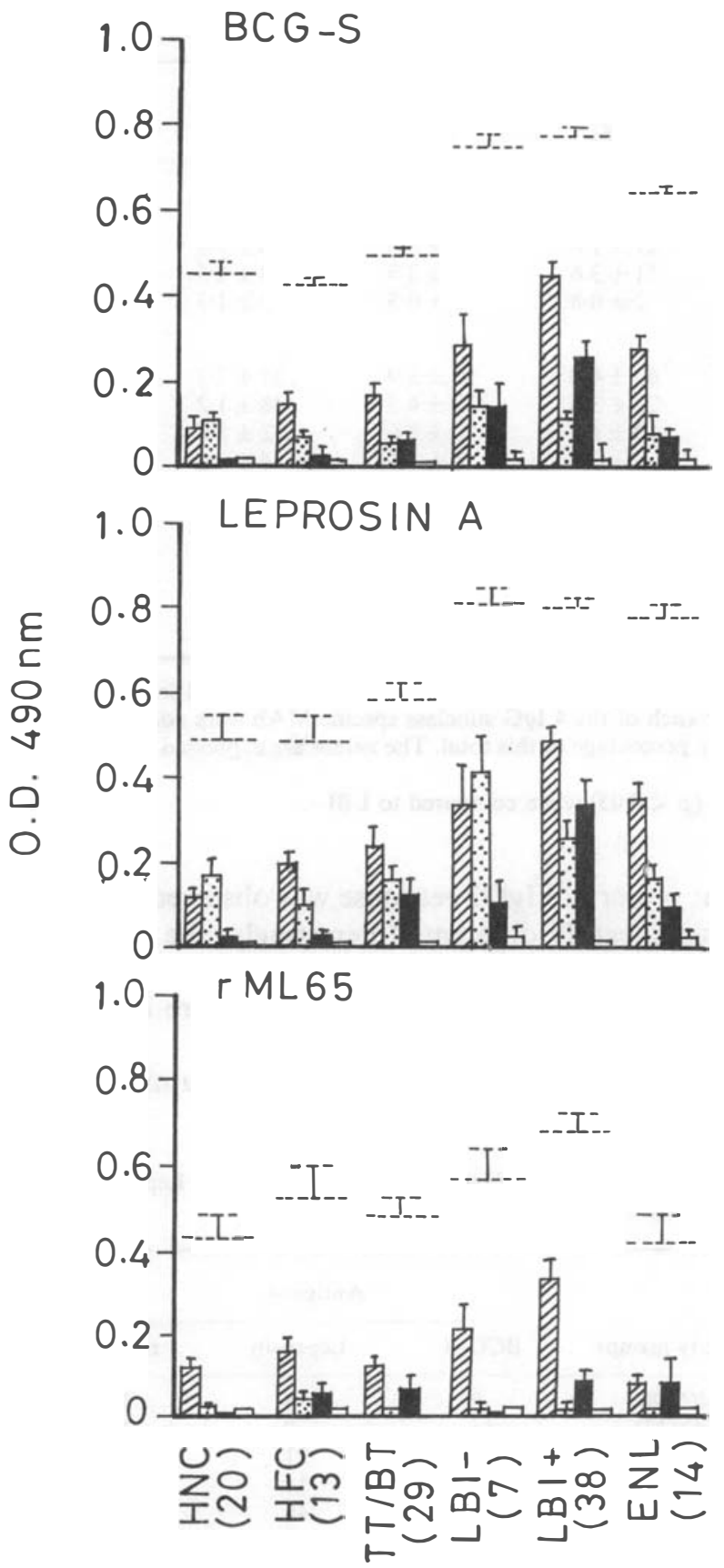

Figure 1. IgG subclass responses of leprosy patients and healthy contacts and noncontacts to BCG-S, leprosin $\mathrm{A}$ and rML65 antigens. ELISA plates were coated with BCG-S, leprosin A or rML65 $(4 \mu \mathrm{g} / \mathrm{ml})$ and incubated with $1: 20$ dilution of serum samples. Antihuman IgG subclass MAb (ख্Z, IgG1;, $\operatorname{IgG} 2 ; \square, \operatorname{IgG} 3 ; \square, \operatorname{IgG} 4)$ were added at a dilution of $1: 500$ followed by $1: 2000$ dilution of antimouse IgG-HRP. Parallely, 1 set of plates was treated with antihuman IgG HRP $(1: 1000)$ after incubation with the serum samples to measure the IgG levels (dashed lines). The number of serum samples tested for each group is given in parentheses. 
Table 1. Relative proportions of IgG subclass responses to BCG-S, leprosin A and rML65

\begin{tabular}{|c|c|c|c|c|c|c|}
\hline & $\begin{array}{c}\text { HNC } \\
{[20]}\end{array}$ & $\begin{array}{r}\mathrm{HFC} \\
{[13]}\end{array}$ & $\begin{array}{c}\text { TT/BT } \\
\text { [29] }\end{array}$ & $\begin{array}{c}\text { LBI- } \\
\text { [7] }\end{array}$ & $\underset{[38]}{\mathrm{LBI}+}$ & $\begin{array}{c}\text { ENL } \\
{[14]}\end{array}$ \\
\hline \multicolumn{7}{|l|}{ BCG-S } \\
\hline IgG1 & $40 \pm 4 \cdot 2$ & $60 \pm 3 \cdot 5$ & $55 \pm 3 \cdot 5$ & $49 \pm 3 \cdot 2$ & $55 \pm 2 \cdot 8$ & $60 \pm 3 \cdot 6$ \\
\hline IgG2 & $52 \pm 4.9$ & $27 \pm 1 \cdot 8$ & $24 \pm 3 \cdot 5$ & $30 \pm 7 \cdot 9$ & $18 \pm 2.2$ & $21 \pm 3 \cdot 6$ \\
\hline IgG3 & $5 \pm 1 \cdot 4$ & $11 \pm 3.6$ & $18 \pm 3 \cdot 5$ & $18 \pm 6 \cdot 5$ & $26 \pm 2 \cdot 8$ & $15 \pm 3 \cdot 5$ \\
\hline IgG4 & $3 \pm 0.7$ & $2 \pm 0.8$ & $3 \pm 0 \cdot 5$ & $3 \pm 1 \cdot 7$ & $2 \pm 0 \cdot 3$ & $4 \pm 0.5$ \\
\hline \multicolumn{7}{|c|}{ leprosin A } \\
\hline IgG1 & $39 \pm 4 \cdot 8$ & $60 \pm 4 \cdot 4$ & $50 \pm 3 \cdot 9$ & $37 \pm 7 \cdot 2$ & $49 \pm 3 \cdot 5$ & $54 \pm 6 \cdot 5$ \\
\hline IgG2 & $50 \pm 5 \cdot 5$ & $29 \pm 3.6$ & $33 \pm 4 \cdot 5$ & $48 \pm 1 \cdot 2$ & $24 \pm 3.1$ & $25 \pm 4.9$ \\
\hline IgG3 & $8 \pm 3 \cdot 0$ & $10 \pm 1.9$ & $18 \pm 3.6$ & $12 \pm 5 \cdot 8$ & $25 \pm 3 \cdot 3^{*}$ & $16 \pm 4.9$ \\
\hline IgG4 & $3 \pm 0.9$ & $3 \pm 1 \cdot 1$ & $4 \pm 1 \cdot 6$ & $4 \pm 1 \cdot 6$ & $2 \pm 0.5$ & $5 \pm 0.8$ \\
\hline \multicolumn{7}{|l|}{ rML65 } \\
\hline IgG1 & $75 \pm 5 \cdot 6$ & $65 \pm 6 \cdot 1$ & $72 \pm 5 \cdot 0$ & $86 \pm 5 \cdot 8$ & $73 \pm 4 \cdot 2$ & $47 \pm 7 \cdot 5$ \\
\hline IgG2 & $16 \pm 3 \cdot 7$ & $17 \pm 4 \cdot 3$ & $9 \pm 1 \cdot 8$ & $5 \pm 2 \cdot 9$ & $8 \pm 2.3$ & $22 \pm 7 \cdot 3$ \\
\hline IgG3 & $4 \pm 2 \cdot 0$ & $14 \pm 5 \cdot 6$ & $19 \pm 4 \cdot 3$ & $5 \pm 1 \cdot 8$ & $16 \pm 3 \cdot 2^{*}$ & $15 \pm 6.9$ \\
\hline IgG4 & $6 \pm 3.7$ & $5 \pm 2 \cdot 6$ & $1 \pm 0.4$ & $5 \pm 3 \cdot 4$ & $4 \pm 1 \cdot 4$ & $15 \pm 6 \cdot 2$ \\
\hline
\end{tabular}

The proportion of IgG subclass response was calculated as described in ref. 23. The absorbance values (presented in Figure 1) for each of the $4 \mathrm{IgG}$ subclass specific MAb were added and individual IgG subclass response was expressed as a percentage of this total. The values are expressed as mean $\pm \mathrm{SE}$ of the percentage response.

*Significantly different $(p<0.05)$ when compared to LBI-.

IgG2 subclass. Similar pattern of IgG3 response was observed for BCG-S, even though the levels were not significantly different. Interestingly, the subclass pattern of antileprosin A and antiBCG-S IgG antibodies in ENL patients was similar to that of TT/BT group (Figure 1, Table 1), even though ENL reactions are observed in lepromatous patients.

The IgG subclass distribution of antileprosin A and the antiBCG-S response in HFC

Table 2. Proportion of IgG3 responders to BCG-S, Leprosin A and rML65

\begin{tabular}{lccr}
\hline & \multicolumn{3}{c}{ Antigens } \\
\cline { 2 - 4 } Study groups & BCG-S & Leprosin & rML65 \\
\hline HNC (20) & 0 & 5 & 10 \\
HFC (13) & 15 & 0 & 15 \\
TT/BT (29) & 45 & 21 & 31 \\
LBI- (7) & 43 & 14 & 0 \\
LBI + (38) & 68 & 40 & 26 \\
ENL (14) & 36 & 14 & 21 \\
& & & \\
\hline
\end{tabular}

The proportion of individuals among the various study groups with IgG3 antibody levels above the positive cut-off value (mean $+2 \mathrm{SD}$ of $\mathrm{HNC}$ ) is given.

The number of serum samples tested for each group is given in parentheses. 
was different from HNC, even though their IgG levels to these antigens were comparable (Figure 1). In HFC, IgG1 response was predominant, while HNC showed equivalent proportion of IgG1 and IgG2 antibodies to leprosin A and BCG-S (Table 1).

Minor differences were also observed in the IgG subclass pattern in the anti-rML65 response. However, IgG1 was the predominant subclass in the rML65 response in all the groups of patients and controls. ENL patients had equivalent levels of IgG1 and IgG3 (Table 1). On the other hand, antibodies of IgG4 subclass against the 3 antigens studied were at low levels in all the groups (Figure 1).

\section{Discussion}

The $4 \mathrm{IgG}$ subclasses have distinct effector functions. While IgG1 and IgG3 are efficient in fixing complement and mediating ADCC, IgG2 can mediate opsonization only at very high epitope densities. ${ }^{10}$ On the basis of effector functions, the importance of IgG2 subclass in the clearance of capsulated microorganisms is well documented. ${ }^{24}$ However, the subclass antibody responses in leprosy has not been addressed in detail. In the leprosy spectrum, despite the high levels of $M$. leprae specific IgG antibodies in the lepromatous patients, the bacterial load remains high. ${ }^{1}$ In this study, an attempt was made to analyse the subclass distribution of the antimycobacterial antibodies in a leprosy endemic population. With the availability of purified recombinant proteins of $M$. leprae and defined MAb reagents to human IgG subclasses, ${ }^{21,22}$ it is now possible to address the questions on the subclass responses.

In accordance with published reports, IgG antibodies to sonicated antigens of M. leprae and BCG were higher in lepromatous patients than in all other groups. However, in this study, the levels were the same in both BI positive and negative lepromatous patients (Figure 1). In a larger study, ${ }^{25}$ serum IgG antibodies to $M$. leprae sonicate were at significantly higher levels in LBI+ than in LBI- patients. The observed difference may be either due to the variations in the antigenic composition of leprosin $\mathrm{A}$ used ${ }^{26}$ or due to the fewer LBI- cases studied here. The salient observation of this study is the difference in the pattern of IgG subclass responses between different groups of patients and contacts. Assuming that proteins are the predominant antigens in the sonicate preparations of BCG and M. leprae, the IgG3 responses were elevated both in quantity and proportion, in the LBI+ group when compared to LBI - group. On the other hand, in the LBI- patients, IgG2 responses were dominant (Figure 1, Table 1). Since the antigens are sonicate preparations, the differences in the subclass responses suggest two possibilities. (A) There is a switch in the subclass responses to the same antigens during different phases of the disease; or (B) different antigens are immunodominant during the different phases of the disease.

The first possibility was tested by analysing the IgG subclass antibody levels to purified recombinant proteins of $M$. leprae. It has already been demonstrated that the $\mathrm{IgG}$ response to purified recombinant $65 \mathrm{kDa}$ of $M$. leprae and $M$. bovis and $70 \mathrm{kDa}$ of M. tuberculosis was significantly decreased in LBI- patients when compared to LBI+ patients (Figure 1 and ref. 27). In this study it was observed that IgG1 was the dominant subclass in response to rML65 in all the groups studied, suggesting that any given antigen preferentially elicits only one type of subclass antibodies in leprosy. Moreover, LAM has been shown to elicit predominantly $\operatorname{IgG} 2$ response. ${ }^{9}$ Together, these observations indicate that the second suggestion may be more relevant. Analysis of 
subclass antibodies to other purified proteins are underway to understand more about the pattern of subclass response and its relevance to the disease process.

The observed increases in the level of IgG3 antibodies to leprosin A in LBI+ patients when compared to LBI - and tuberculoid patients in this study are contrary to the findings of Dhandayuthapani et al., ${ }^{9}$ wherein $\operatorname{IgG} 2$ was the predominant subclass to leprosin A over and above the IgG1 levels. Based on this observation, the authors concluded that most of the IgG subclass responses to leprosin A could be accounted for by the reactivity with lipoarabinomannan, a carbohydrate antigen which elicited a predominantly IgG2 response. This discrepancy may well be due to their use of a monoclonal (SG-16) to IgG1 proteins of $\mathrm{Glm}(\mathrm{f})$ allotype which is represented at low frequency in the study population. ${ }^{28}$ However, in the present study, based on the presence of $\mathrm{IgG} 1$ and $\mathrm{IgG} 3$ responses, it is clear that apart from LAM, other dominant protein antigens are also present in leprosin A.

In lepromatous patients undergoing ENL reactions, the pattern of IgG subclass response observed was similar to that of tuberculoid patients. Their anti-rML65 IgG, especially IgG1 levels, were decreased significantly. We do not know the basis for the observed differences. A longitudinal study of lepromatous patients undergoing reactions may help in correlating the lymphokine patterns ${ }^{29,30}$ with IgG subclass responses. However, these patients do not have elevated levels of $\operatorname{IgG} 4$, even though the antiIgG4 monoclonal antibody reagent used in this study has high affinity. ${ }^{31}$

Another salient finding in this study is the difference in the pattern of subclass response among healthy family contacts and endemic controls (HNC). In fact, IgG subclass pattern of HFC was more similar to tuberculoid patients than to HNC. The specific increase in the proportion of IgG1 and IgG3 in HFC (Table 1) suggests that their immune system is primed selectively to mycobacterial protein antigens. In accordance with our other studies in the same population, ${ }^{27}$ the immune profile against mycobacterial antigens is specifically altered in HFC even though both groups of healthy controls are derived from the same endemic area.

The elevated IgG antibody response to rML65 in lepromatous patients was dominated by IgG1 and to a lesser extent by IgG3 (Figure 1), which are the predominant isotypes expected in response to protein antigens. ${ }^{11,12}$ It is of interest to note that both IgG1 and IgG3 levels to rML65 were reduced in LBI- patients and IgG1 in ENL patients. At present the meaning of these alterations in subclass pattern is not clear and neither do we understand the functional significance of $\mathrm{IgG}$ subclass pattern in relation to pathogenesis. However, it is possible that certain regulatory mechanisms play a crucial role in lepromatous leprosy. Such a regulatory mechanism is even more relevant because of our recent finding of an inverse relationship between cell mediated and humoral immunity of rML65 in both leprosy patients and endemic controls. ${ }^{32}$

Recent studies have demonstrated distinct patterns of expression of cytokines in lepromatous and tuberculoid patients in vitro, thereby implicating a role for $\mathrm{T}$-cell subsets in the immune spectrum of leprosy. ${ }^{29}$ The regulatory role of cytokines in the class switching of immunoglobulin isotypes is well documented in the murine system but not in humans. ${ }^{18}$ However, recent studies by Kitani \& Strober ${ }^{19}$ have shown that the various human IgG subclasses manif est distinct requirements of cytokines for the regulation of early steps in isotype differentiation. Further analysis of the pattern of $\mathrm{IgG}$ isotypes induced in response to purified mycobacterial antigens may help in understanding the dichotomy of the immune response observed in leprosy. 
Yamamura et al. ${ }^{29,30}$ have demonstrated the differences in the expression of $\mathrm{T}$ helper subsets in leprosy across the spectrum with the expression of Th2-like pattern of cytokines in the lesions of lepromatous leprosy patients. However, no overt increase in IgG4 was observed in these patients, unlike in parasitic infections ${ }^{16,17}$ wherein elevated IL-4 has been shown to be associated with IgG4 and IgE. While the regulation of IgE and IgG4 by IL-4 is well documented in humans, the regulation of other subclasses is not clear. Studies on the expression of IgG subclass germline transcripts ${ }^{19}$ have revealed that in the presence of a proliferative stimulus IgG3 in augmented by IL-4 and IgG1 by IL-2, while cytokines are sufficient for the induction of IgG1 and IgG4 (by IFN- $\gamma$ and IL-4, respectively). Therefore, it is possible that induction of $\mathrm{IgG} 3$ in lepromatous leprosy patients may be accomplished specifically by Th2-like cells induced by certain antigens of $M$. leprae, and that it is downregulated following chemotherapy. It appears that such a modulation is brought about more specifically in response to $M$. leprae, since we did not observe any difference between patients and controls in the IgG subclass pattern to several autoantigens (S. Ilangumaran and R. Sheela, unpublished observations).

It is well documented that in the highly bacilliferous lepromatous leprosy patients effective cell-mediated immune response is not mounted, partially due to the absence of IL-2 and IFN- $\gamma$. However, antigen specific $\mathrm{T}$ cell help to B cell is present in order to account for the high levels of IgG, especially IgG1 and IgG3 subclasses to protein antigens. With more knowledge on the regulation of $\mathrm{IgG}$ subclasses in humans, it should be possible to understand the regulation of the antibody response by $\mathrm{T}$-cell subsets in leprosy.

\section{Acknowledgments}

This work was supported by grants from Department of Biotechnology, Government of India and Indo-Swiss Co-operation in Biotechnology, Zurich, Switzerland. R.S. thanks CSIR, Government of India for financial assistance in the form of junior and senior research fellowships.

\section{References}

1 Godal T. Immunological aspects of leprosy/Present status. Prog Aller, 1978; 25: 211-42.

2 Bloom BR, Mehra V. Immunological unresponsiveness in leprosy. Immunol Rev, 1984; 80: 5-28.

3 Kaplan G, Cohn ZA. Leprosy and cell mediated immunity. Current Opinion in Immunol, 1991 ; 3: 91-6.

${ }^{4}$ Lim SD, Fusaro RM. Leprosy IV. The quantitation of immunoglobulins (IgG, IgA and IgM) in leprosy sera. Int J Lepr, 1968; 36: 144-53.

5 Sengupta U, Sinha S, Ramu G. Immunological assessment of sera of leprosy patient. Lepr India, 1979; 51: 43-8.

6 Choudhary MS, Singh NB, Srivatsa K, Gupta HP. Patterns of immunoglobulins in the serum of leprosy patients. Ind J Lepr, 1987; 59: 178-87.

7 Melsom R, Harboe M, Myrvang B, Godal T, Belehu A. Immunoglobulin class specific antibodies to $M$. leprae in leprosy patients, including the indeterminate groups and healthy contacts as a step in the development of methods for serodiagnosis of leprosy. Clin Exp Immunol, 1982; 47: 225-33.

8 Touw J, Langendijk EM, Stoner GL, Belehu A. Humoral immunity in leprosy: immunoglobulin G and M antibody responses to Mycobacterium leprae in relation to various disease patterns. Infect Immun, 1982; 36: 885-92.

9 Dhandayuthapani S, Izumi S, Anandan D, Bhatia, VN. Specificity of IgG subclass antibodies in different clinical manifestations of leprosy. Clin Exp Immunol, 1982; 88: 253-257. 
10 Jefferis R, Kumararatne DS. Selective IgG subclass deficiency: quantification and clinical relevance. Clin Exp Immunol, 1990; 81: 357-67.

11 Skvaril F. IgG subclasses in viral infections. Monogr Allergy, 1986; 19: 134-43.

12 Hammerstrom L, Smith CIE. IgG subclass changes in response to vaccination. Mongr Allergy, 1986; 19: $122-33$.

13 Bruggemann M, Williams GT, Bindon CI, Clark MR, Walker MR, Jefferis R, Waldman H, Neuberger MS. Comparison of the effector functions of human immunoglobulins using a matched set of chimeric antibodies. J Exp Med, 1987; 166: 1351-61.

14 Linde GA, Hammarstrom L, Person MAA, Smith CI, Sunqvist VA, Wahren B. Virus specific antibody activity of different subclasses of immunoglobulins $\mathrm{G}$ and $\mathrm{A}$ in cytomegalovirus infections. Infect Immun, 1983; 42: 237-44.

15 Ljunggren K, Boliden PA, Mansson LM, Jondal M, Wahren B. IgG subclass response to HIV in relation to antibody dependent cellular cytotoxicity at different clinical stages. Clin Exp Immunol, 1988; 73: 343-7.

16 Hussain R, Grogl M, Ottesen EA. IgG antibodies subclass in human filariasis. Differential subclass recognition of parasitic antigens correlates with different clinical manifestations of infections. J Immunol, 1987; 139: 2794-8.

17 Cabrera Z, Buttner DW, Parkhouse RME. Unique recognition of a low molecular weight Onchocerca volvulus antigen by IgG3 antibodies in chronic hyperreactive onchodermatitis (Sowda). Clin Exp Immunol, 1988; 74: 223-9.

18 Snapper CM, Mond JJ. Towards a comprehensive view of immunoglobulin class switching. Immunol Today, 1993; 14: 15-17.

19 Kitani A, Strober W. Regulation of $\mathrm{C} \gamma$ subclass germ-like transcripts in human peripheral blood B cells. J Immunol, 1993; 151: 3478-88.

20 Ridley DS, Jopling WH. Classification of leprosy according to immunity. A five group system. Int J Lepr, 1966; 34: 255-73.

21 Jefferis R, Reimer CB, Skvani F, de Lange G, et al., Evaluation of monoclonal antibodies having specificity for human IgG sub-classes: results of an IUIS/WHO collaborative study. Immunol Lett, 1985; 10: $223-52$.

22 Jefferis R, Reimer CB, Skvani F, de Lange G, et al., Evaluation of monoclonal atnibodies having specificity for human IgG subclasses: results of the 2nd IUIS/WHO collaborative study. Immunol Lett, 1992; 31: 14368.

${ }^{23}$ Loizou S, Cofiner C, Weetman AP, Walport MJ. Immunoglobulin class and IgG subclass distribution of anticardiolipin antibodies in patients with systemic lupus erythematosus and associated disorders. Clin Exp Immunol, 1992; 90: 434.

24 Burton DR, Woof JM. Human antibody effector functions. Adv Immunol, 1992; 51: 1-84.

25 Mathew JM, Muthukkaruppan VR. Class specific immunoglobulins and antibodies to mycobacterial sonicates and autoantigens in leprosy patients and contacts. Microbial Pathogen, 1993; 15: 367-76.

26 Ibrahim MA, Lamb FI, Colston MJ. Analysis of variation in batches of Armadillo derived Mycobacterium leprae by immunoblotting. Int J Le pr, 1990; 58: 73-7.

27 Ilangumaran S, Shanker Narayan NP, Ramu G, Muthukkaruppan VR. Antibody response to recombinant $65 \mathrm{kDa}, 70 \mathrm{kDa}$ and $18 \mathrm{kDa}$ mycobacterial antigens in leprosy patients and healthy contacts in a leprosy endemic population. Int J Lepr, 1994; in press.

28 Jefferis R. Standardization: what wants it? Clin Exp Immunol, 1992; 89: 500-1.

29 Yamamura M, Uyemura K, Deans RJ, Weinberg K, Rea TH, Bloom BR, Modlin RL. Defining protective responses to pathogens: cytokine in profiles in leprosy skin lesions. Science, 1991; 254: 277-9.

30 Yamamura M, Wang XH, Ohmen JD, Uyemura K, Rea TH, Bloom BR, Modlin RL. Cytokine patterns of immunologically mediated tissue damage. J Immunol, 1992; 149: 1470-5.

31 Barra A, Schulz D, Aucoturier P, Preud'homme J-L. Measurement of anti-Haemophilus influenzae type b capsular polysaccharide antibodies by ELISA. J Immunol Methods, 1988; 115: 111-7.

32 Ilangumaran S, Shanker Narayan NP, Ramu G, Muthukkaruppan VR. Cellular and humoral immune responses to recombinant $65 \mathrm{kDa}$ antigen of Mycobacterium leprae in leprosy patients and healthy contacts in a leprosy endemic population. Clin Exp Immunol, 1994; 96: 79-85. 